\title{
Quality Palliative Care or Physician-assisted Death: A Comment on the French Perspective of End-of-life Care in Neurological Disorders
}

\author{
Mohamed Y. Rady ${ }^{1 *}$, Joseph L. Verheijde ${ }^{2}$ and Michael Potts ${ }^{3}$ \\ ${ }^{1}$ Professor of Medicine, Department of Critical Care Medicine, Mayo Clinic, Phoenix, Arizona, Center for Biology and Society School of Life Sciences, Arizona State \\ University, Tempe, Arizona, USA \\ ${ }^{2}$ Associate Professor of Biomedical Ethics, Department of Physical Medicine and Rehabilitation, Mayo Clinic, Phoenix, Arizona Center for Biology and Society School of \\ Life Sciences, Arizona State University, Tempe, Arizona, USA \\ ${ }^{3}$ Professor of Philosophy, Department of philosophy and religion, Methodist University, Fayetteville, North Carolina, USA
}

\begin{abstract}
Conflating physician-assisted death with palliative care is a growing concern in medicine. Palliative care is symptommanagement medical care without actively shortening the end-of-life trajectory of a terminal illness. Physician-assisted death intentionally shortens the dying process to bring about preplanned death as the means of relieving suffering. Physician-assisted death may be conflated with palliative care where this practice is illegal, eg, France. The Revised French Code of Medical Ethics states that when the decision to withdraw or withhold treatment is made in accordance with applicable law, and even if the patient has brain damage precluding an assessment of suffering, physicians must use the treatments, including analgesics and sedatives, to maximize the quality of end of life, safeguard the patient's dignity, and comfort relatives. This revision, which in France has the force of law, appears to uphold the Hippocratic Oath to alleviate suffering and deliver compassionate care. Additional analysis raises questions: (1) what type of treatment is being withdrawn or withheld? (2) What type of brain damage or neurological disability might preclude the assessment of suffering? (3) What type of suffering (eg, physical, psychosocial, existential, etc.) must be treated? (4) What measure of proportionality is applicable to ensure that sedatives and analgesics will not be the proximate causation of death? The legislation assumes potential suffering from treatment withdrawal which disrupts the current ethical paradigm on withdrawing versus withholding treatment. This legislation also applies to neurologically disabled patients incapable of requesting euthanasia but for whom a treatment limitation decision has been made. Re-evaluation of the double-effect principle, intention, and causation of death precludes using the term palliative care. The two-step process (ie, treatment withdrawal and administration of sedatives and analgesics) should be considered physician-assisted death in some neurologically disabled persons. The revision implicitly paves the way to organ donation euthanasia in neurological disorders.
\end{abstract}

Keywords: Neurological disorders; Dying; End-of-life care; Euthanasia; Legislations; Non-heart-beating; Donation; Palliation; Physician-assisted death; Sedation

\section{Introduction}

There are growing concerns in the medical community about conflating physician-assisted death (PAD) with palliative care in endof-life (EOL) care [1]. Palliative care is symptom-management medical care without active intervention in the EOL trajectory of a terminal illness. PAD includes intentional life-ending acts that accelerate the dying process to bring about a preplanned death as the means of relieving suffering [2]. Physician-assisted suicide is legal in some European countries (the Netherlands, Belgium and Switzerland) and two states (Oregon and Washington) in the United States [2,3]. Euthanasia is legalized in the Netherlands, Luxemburg and Belgium. PAD collectively refers to either euthanasia or physician-assisted suicide. PAD can be voluntary (with the patient's knowledge and consent), nonvoluntary (without the patient's consent) and involuntary (against the patient's wishes). PAD may be conflated with providing appropriate palliative care services in societies and countries in which euthanasia is illegal eg, France. Conflating PAD with palliative care can avoid the need for an open debate about its legal and ethical permissibility in modern societies with diverse religious affiliations, cultures and ethnicities. Several religious affiliations, cultures and ethnicities in European communities object to life-ending acts and PAD [4].

Baumann and colleagues described a case in France of "a young man in a vegetative state whose gastric tube was withdrawn without preliminary sedation [and who] died only 6 days later after experiencing multiple seizures that caused severe distress to the family. This case, together with the Leonetti Mission [A parliamentary mission appointed by the French government to examine quality of EOL care] promoted a revision of Article 37 of the French code of medical ethics [3]". The Revised Article 37 and Article 38 text is the English translation from Baumann and colleagues with emphasis added [3].

The new revision authorizes the use of analgesics and sedatives to alleviate suffering caused by treatment withdrawal or withholding in patients with brain damage [3]. It applies to both minors and adults. The legislation is not specific on what constitutes "brain damage that precludes an assessment of his or her suffering" nor does it specify the kind of treatment that is being withdrawn or withheld. The terms "brain damage" and "preclude an assessment of suffering" are sufficiently vague to permit the inclusion of patients (minors and adults) with a

${ }^{*}$ Corresponding author: Mohamed Y. Rady, Professor of Medicine, Department of Critical Care Medicine, Mayo Clinic, Phoenix, Arizona, Center for Biology and Society School of Life Sciences, Arizona State University, Tempe, Arizona, USA, E-mail: Rady.mohamed@mayo.edu

Received February 27, 2011; Accepted March 25, 2011; Published March 27, 2011

Citation: Rady MY, Verheijde JL, Potts M (2011) Quality Palliative Care or Physician-assisted Death: A Comment on the French Perspective of End-of-life Care in Neurological Disorders. J Clinic Res Bioeth 2:102e. doi:10.4172/21559627.1000102e

Copyright: (C 2011 Rady MY, et al. This is an open-access article distributed under the terms of the Creative Commons Attribution License, which permits unrestricted use, distribution, and reproduction in any medium, provided the original author and source are credited. 
broad spectrum of neurological disorders affecting the central nervous system (ie, the whole brain and spinal cord). Brain damage includes, but is not limited to, chronic congenital and acquired neurodegenerative disorders (eg, dementia), impaired states of consciousness (eg, minimal conscious state, vegetative state, locked-in-syndrome etc,), and neurological disorders resulting in cognitive, physical, and neuropsychiatric disabilities, which are all frequently associated with the assessment of "poor quality of life" for the patient. The term "brain damage" also encompasses acute brain or spinal cord injury in minors and adults resulting from trauma, hypoxia or ischemia.

\section{Revised Article 37 of the French Code of Medical Ethics (Article R. 4127-37 of the French Code of Public Health)}

"III - When treatment withdrawal or withholding is decided in accordance with Article L.1110-5 and Articles L1111-4 and L1111-

13 , under the conditions considered in paragraphs I and II of the present article, and even if the patient has brain damage that precludes an assessment of his or her suffering, the physician must use the treatments, including analgesics and sedatives, designed to accompany the patient in compliance with the principles, and under the conditions, stated in Article R4127-38. The physician must also make sure that the patient's relatives are informed of the situation and receive the support they need [3]".

\section{Article 38 of the French Code of Medical Ethics (Article R.4127- 38 of the French Public Health Code)}

"The physician must accompany the dying patient until the very last moment, provide appropriate care and other measures to maximize the quality of the end of life, safeguard the dignity of the patient, and comfort the relatives. The physician does not have the right to intentionally cause death [3]".

Section I of the Revised Article 37 (French Code of Medical Ethics) mentions that physicians "may refrain from starting or continuing treatments that appear pointless or disproportionate or that have no other aim or effect than the artificial maintenance of life [3]." Permanent treatment for artificial maintenance of life may be required only in a small subset of patients with brain damage. If temporary life-sustaining treatment is not withdrawn too early during the initial phase of critical illness and physiological instability after acute severe brain injury, most patients with neurological disabilities can survive without permanent dependency on artificial means to maintain life [5]. The usual life-sustaining treatment for patients who are unable to eat and drink is artificial nutrition and hydration (ANH) support. It is rare that brain damage patients require permanent respiratory support with mechanical ventilation unless they suffer from permanent damage of the respiratory centers in the brainstem or the spinal cord segment controlling the respiratory muscles (eg, quadriplegia). The term of withholding of treatment can include patients who voluntarily stopped eating and drinking because of their neurological disorders.

In this editorial, we will use withdrawal of life-support treatment (WLST) in some patients with neurological disorders to signify the range of treatment withdrawal or withholding: voluntarily stopping eating and drinking, $\mathrm{ANH}$, and respiratory support (in those who are totally dependent on mechanical ventilation). Panksepp et al have previously argued that WLST from patients in a vegetative state can inflict pain and suffering [6]. Pain, consciousness and suffering are difficult to assess in some patients with brain damage after WLST [3]. We outline how the ambiguity of terms such as 'brain damage,' 'treatment withdrawal or withholding', and the phrase 'use of the treatments, including analgesics and sedatives' can be construed as permitting PAD (euthanasia) in the French legislation. The French model also applies to neurologically disabled patients "incapable of asking for euthanasia but in whom a treatment limitation decision has been made [3]."

\section{Sedatives and EOL care}

Sedatives are used as part of normal EOL care. Quill and colleagues proposed the use of the following terms: (1) 'proportionate palliative sedation' when using the minimum amount of sedation necessary to relieve refractory physical symptoms at EOL, and, (2) 'palliative sedation' when the intended end point is induction of unconsciousness [7]. In two types, the physical symptoms or depth of unconsciousness can be continuously assessed in patients. We have previously distinguished two types of sedation practices in EOL care: (1) normal sedation practice (or proportionate sedation), and (2) continuous deep sedation (or terminal sedation) [1]. However, a third category of palliative sedation called "early terminal sedation" has been described in patients who wish to die [8]. Cellarius described "early terminal sedation as a practice composed of two legally and ethically accepted treatment options. Under certain conditions, patients have the right to reject hydration and nutrition, even if these are life-sustaining. Patients are also entitled to sedation as palliation for intolerable, intractable suffering" [8]. We postulate that the use of sedatives and analgesics as outlined in the French Code of Medical Ethics cannot always be justified on the ground of proportionate sedation. We will discuss several reasons why the use of these drugs may be more appropriately characterized as continuous deep sedation or early terminal sedation.

First, Baumann and colleagues accept the difficulty of measuring or objectively assessing the severity of pain and level of consciousness in some patients with brain damage [3]. In clinical practice, an appropriate assessment of the proportionality of sedation is not feasible in situations in which the symptoms that are to be controlled cannot be measured or assessed accurately. The proportionality of medication effects in neurological disorders is indirectly assessed by the patient's moaning, groaning or by increases in vital signs, eg, heart rate, respiration and blood pressure [3]. In addition, the legislative use of the term "suffering" does not limit its applicability to suffering from physical symptoms only but also leaves open its use in the context of suffering from psychosocial and existential distress.

Second, the pharmacokinetics and pharmacodynamics of sedatives and analgesics vary in the final phase of dying. The proportionality of the pharmacological dose-response in each patient becomes a relevant factor when titrating medications to distinguish palliating symptoms from accelerating the dying process [1]. For instance, in the final phase of dying, delayed or impaired drug clearance and worsening encephalopathy from multiple failing organs influence pharmacological effects of sedatives and opioids. Administering fixed or escalating doses of these medications without careful attention to drug clearance and brain function can cause medication overdose and contravene the proportionality of sedation. As a result, large cumulative doses of benzodiazepines and opioids may be given to moribund patients before death [9]. When opioids and benzodiazepines are given in excess of actual patient's needs, some commentators questioned whether these are "given to treat the pain and anxiety of the patient's family, nurses, and doctors" [10]. An international survey of the variability of EOL use of sedatives and analgesics in neonatal care units suggested that these medications were given and titrated in physiologically stable infants with permanent neurological impairment to comfort the parents and/or the health care providers [11]. Physicians have a moral and professional 
responsibility to be aware of these facts so that they can avoid giving care that is not truly palliative and which can be the proximate cause of the patient's death.

Third, in palliative care, opioids are the preferred analgesics to relieve pain and benzodiazepines are the sedatives of choice to relieve anxiety [1]. Clinically, it may be difficult to distinguish between these two symptoms. However, benzodiazepines are poor analgesics, and opioids are poor anxiolytics. For example, titrating sedatives instead of analgesics for the control of pain can induce unconsciousness without pain relief and, in higher doses, induce deep coma and depress brainstem reflexes including vital signs [12]. On the other hand, titrating analgesics instead of sedatives for the control of anxiety can induce narcosis from carbon dioxide build up from respiratory depression or apnea without relieving anxiety.

\section{Conflating PAD with palliative care}

Baumann and colleagues described the new French legislation which states that "When a decision is made to withdraw treatments intended to prolong life, the physician must bear in mind that the law authorizes the withdrawal of such treatments only with the condition that any additional suffering potentially caused by treatment withdrawal will be completely eliminated by palliative care [3]" [emphasis added]. We argue that the two-step process (ie, WLST and administration of sedatives and analgesics) in some neurologically disabled persons conflates PAD with palliative care. Further analysis of the double-effect principle, intention, and causation of death (outlined below) will show that using the term 'palliative care' in this context is inappropriate.

\section{The double-effect principle in EOL care}

The double-effect principle is often invoked to justify physicians' acts in EOL care that can be morally challenging [1]. This principle justifies the administering of opioids to control pain (benefit to the patient) even though this medication could cause the foreseeable hastening of death (harm to the patient) from respiratory depression. However, the double-effect principle may be misused for characterizing intentional harm as unwanted foreseeable harm from life-ending interventions [1]. The Revised French Code of Medical Ethics describes a twointervention process: the first intervention is WLST which is coupled with the second intervention of administering analgesics and sedatives [3]. However, this legislation anticipates that the intervention of WLST (intended not to artificially prolong life) will also cause harm (pain and suffering) in a neurologically disabled patient before an inevitable death. In this particular situation, life-support treatment is withdrawn because of a decision that the patient's quality of life is unworthy of maintaining, although the treatment is effective and successful in maintaining patient's life. The question is then: why would a physician intentionally perform such an intervention (ie, WLST)? Using the double-effect principle, it may be argued that pain and suffering is an unwanted foreseeable harm but not an intended harm from WLST. However, since WLST can result in harm (pain and suffering) without an outweighing benefit (unless not prolonging life is the desired benefit) in a neurologically disabled patient, then the double-effect principle can no longer morally justify this intervention. Panksepp and colleagues addressed this exact point when discussing WLST in patients in vegetative state: "If so, withdrawal of life-support may violate the principle of nonmaleficence and be tantamount to inflicting inadvertent cruel and unusual punishment on patients whose potential distress, during the process of dying, needs to be considered in ethical decision-making about how such individuals should be treated, especially when their lives are ended by termination of life-supports" [6].
Of course, we agree with Baumann et al that the use of sedatives and analgesics to alleviate suffering is an ethical obligation and a duty of the physician to his (her) patient. However, the suffering is the consequence of an intentional life-ending intervention and its relief constitutes euthanasia (an ancient Greek word meaning good death) or PAD. The French legislation assumes suffering from treatment withdrawal which creates a new ethical paradigm on the moral equivalency of withdrawal and withholding treatment in patients with neurological disorders. Panksepp and colleagues concluded that terminating the lives of persons with chronic disorders of consciousness "...may dictate the use of more rapid pharmacological forms of euthanasia that minimize distress [rather] than the de facto euthanasia of life-support termination that may lead to excruciating feelings of pure thirst and other negative affective feelings in the absence of any reflective awareness [6]".

\section{Intention and causation of death}

Intention and proximate causation of death can distinguish PAD from palliative care [13]. The French legislation describes a two-step process in physician's acts: the first act is WLST and the second one is administering analgesics and sedatives [3]. If the first act is not performed then the second act may not be needed. If neither acts take place, patients with brain damage still die but at unpredictable EOL trajectories. The intention of the first act (WLST) is not to prolong life (or hasten death). The second act of administering sedatives and analgesics is intended to alleviate suffering from the initial act. Sprung et al described EOL interventions intended to hasten death as the means of relieving suffering that could be confused with palliative care [14]. The two-step process is consistent with planning an elective death with reasonable predictable EOL trajectory. Medical interventions that intentionally shorten the dying process do not fall within the practice of palliative care, but perhaps should be recognized as PAD $[1,14]$. Baumann and colleagues denoted this as a "scheduled" death [3].

At this point it is reasonable to ask: If the primary intention of the physician is not to cause suffering to a patient with neurological disability or brain damage in the first place, why should he (she) engage in the harmful act of WLST? If WLST causes pain and suffering, then the claim that withdrawing a treatment is medically, ethically or legally equivalent to withholding a treatment is false--in fact, some consider this act as cruel and inconsistent with the Hippocratic practice of medicine [6]. In reality, physicians' thoughts and intentions are private in EOL care and unknown to external observers $[14,15]$; therefore, it becomes guess work to distinguish foreseen vs. intended harm. Quill said in his own words: "multilayered intentions are present in most, if not all, end-of-life decisions... we would do well to look beneath the idealized, sanitized intentions espoused by many medical ethicists to the actual experience of doctors and patients [16]."

\section{Palliative care and medicalization of dying}

The overzealous emphasis on the use of sedatives and analgesics reflects an unsettling societal trend towards the medicalization of dying and death [17]. As Colbert summarized (http://www.uffl.org/ Vol14/colbert-04.pdf): "There are two basic understandings of what it means to be human and a person, and these two concepts underlie two diametrically opposed views on the discipline and practice of medicine, namely, Hippocratic Medicine and the New Medicine...[T] he differences between these two approaches [are seen] in regard to understanding death, dying, suicide and despair, euthanasia, killing and letting die, ordinary and extraordinary care, physician-assisted suicide, advanced directives and living wills, persistent vegetative state, brain and brainstem death, and terminal sedation. A gradual change from the 
Citation: Rady MY, Verheijde JL, Potts M (2011) Quality Palliative Care or Physician-assisted Death: A Comment on the French Perspective of Endof-life Care in Neurological Disorders. J Clinic Res Bioeth 2:102e. doi:10.4172/2155-9627.1000102e

Hippocratic to a "new" model of disease underlies many of the conflicts in ethical and moral decision making in our time [17]."

Comprehensive, high quality palliative care will improve the quality of EOL care for patients and families without hastening death $[18,19]$. Adequate physicians' training and competence in palliative care are key factors to deliver high quality EOL care [20]. For instance, confounding factors such as hypoxia, dehydration, fever etc. can be distressful to patients but are poorly palliated by increasing the doses of sedatives and/or analgesics alone. These confounding factors can be confused with pain or anxiety because they change the same physical signs (increase of heart rate, blood pressure and respiratory rate, moaning and sweating). Specific symptom-control measures and nonpharmacological interventions can alleviate distress much more effectively than simply administering larges doses of sedatives and analgesics [21].

\section{Palliative sedation and organ donation euthanasia}

The Revised Article 37 of the French Code of Medical Ethics has specifically focused on patients with severe neurological disabilities who are also a source of transplantable organs [3]. Organs are procured using non-heart-beating procurement protocols and dependent upon predictable time of death after WLST of less than 90-120 minutes [22]. Organs are procured after 2-5 minutes of loss of arterial pulse or mechanical cardiac asystole. A short time interval from WLST to organ procurement will decrease warm ischemia time and improve organ quality for transplantation. Large cumulative doses of benzodiazepines and opioids have been given to patients who are candidates for nonheart-beating procurement protocols $[9,10]$. Sedatives and analgesics can shorten the time to death and organ procurement after WLST [13]. Organ donation euthanasia is an accepted medical practice in Belgium [23,24]. The same medical practice has also been promoted in other European countries [25]. The separation of palliation from PAD in patients who are candidates for non-heart-beating protocols is both difficult for the patient and difficult to morally justify [13]. Additionally, traditional analgesics and sedatives may not completely blunt awareness and nociception to surgical procurement. Auyong and colleagues reported that non-heart-beating donors had "...changes in processed electroencephalogram when care was withdrawn without the use of hypnotic or anesthetic drugs" [26]. In a correspondence, we recommended that donors should be routinely monitored with electroencephalogram for dosing of anesthetic and opioid drugs during surgical procurement in non-heart-beating protocols [27].

\section{Conclusion}

A careful analysis of the Revised Article 37 French Code of Medical Ethics reveals certain ambiguities and raises several questions: (1) what type of treatment is being withdrawn or withheld? (2) What type of brain damage or neurological disability is precluding assessment of suffering? (3) What type of suffering (eg, physical, psychosocial, existential, etc.) must be treated? (4) What measure of proportionality is applicable to ensure that the administration of sedatives and analgesics is not the proximate causation of death? The new legislation assumes suffering from treatment withdrawal which creates a new ethical paradigm on the moral equivalency of withdrawal and withholding treatment in patients with neurological disorders. This legislation also applies to neurologically disabled patients incapable of requesting euthanasia but for whom a treatment limitation decision has been made. Re-evaluating the double-effect principle, intention, and causation of death in this clinical situation leads us to conclude that using the term palliative care in this particular context is inappropriate. The two-intervention process (ie, treatment withdrawal and administration of sedatives and analgesics) should, in at least some neurologically disabled patients, be considered PAD. As an additional concern, the new French legislation implicitly paves the way to organ donation euthanasia from patients with neurological disabilities who have transplantable organs.

\section{References}

1. Rady MY, Verheijde JL (2010) Continuous Deep Sedation Until Death: Palliation or Physician-Assisted Death? Am J Hosp Palliat Care 27: 205-214.

2. Quill TE (2007) Legal Regulation of Physician-Assisted Death -- The Latest Report Cards. N Engl J Med 356: 1911-1913.

3. Baumann A, Claudot F, Audibert G, Mertes PM, Puybasset L (2011) The ethical and legal aspects of palliative sedation in severely brain injured patients: a French perspective. Philos Ethics Humanit Med 6: 4

4. Sprung C, Maia P, Bulow HH, Ricou B, Armaganidis A, et al. (2007) The importance of religious affiliation and culture on end-of-life decisions in European intensive care units. Intensive Care Med 33: 1732-1739.

5. Wilkinson D (2011) The Window of Opportunity for Treatment Withdrawal. Arch Pediatr Adolesc Med 165: 211-215.

6. Panksepp J, Fuchs T, Garcia VA, Lesiak A (2007) Does any aspect of mind survive brain damage that typically leads to a persistent vegetative state? Ethical considerations. Philos Ethics Humanit Med 2: 32.

7. Quill TE, Lo B, Brock DW, Meisel A (2009) Last-Resort Options for Palliative Sedation. Ann Intern Med 151: 421-424.

8. Cellarius $V(2011)$ 'Early terminal sedation' is a distinct entity. Bioethics 25 46-54.

9. Naim MY, Hoehn KS, Hasz RD, White LS, Helfaer MA, et al. (2008) The Children's Hospital of Philadelphia's experience with donation after cardiac death. Crit Care Med 36: 1729-1733.

10. Finkielman JD (2009) Experience with donation after cardiac death. Crit Care Med 37: 1182.

11. Janvier A, Meadow W, Leuthner SR, Andrews B, Lagatta J, et al. (2011) Whom are We Comforting? An Analysis of Comfort Medications Delivered to Dying Neonates. J Pediatr.

12. Schwartz RS, Brown EN, Lydic R, Schiff ND (2010) General Anesthesia, Sleep and Coma. N Engl J Med 363: 2638-2650.

13. Verheijde JL, Rady MY, McGregor JL (2008) End-of-life care and organ procurement for transplantation: Palliation or euthanasia? Crit Care Med 36: 2481-2482.

14. Sprung CL, Ledoux D, Bulow HH, Lippert A, Wennberg E, et al. (2008) Relieving suffering or intentionally hastening death: Where do you draw the line? Crit Care Med 36: 8-13.

15. Rady MY, Verheijde JL, McGregor JL (2009) Clinical Guidelines and Clinicians' Intentions in End-Of-Life Care. Chest 135: 1696-1697.

16. Quill TE (1993) The Ambiguity of Clinical Intentions. N Engl J Med 329: 10391040.

17. Colbert M (2004) The Medicalization of Death \& Dying. in Life and Learning XIV 227-238.

18. Temel JS, Greer JA, Muzikansky A, Gallagher ER, Admane S, et al. (2010) Early Palliative Care for Patients with Metastatic Non-Small-Cell Lung Cancer. N Engl J Med 363: 733-742.

19. Kelley AS, Meier DE (2010) Palliative Care - A Shifting Paradigm. N Engl J Med 363: 781-782.

20. The Robert H. Lurie Comprehensive Cancer Center at Northwestern University (2011) EndLink: Resource for End of Life Care Education.

21. The EPEC Project Northwestern University's Feinberg School of Medicine (2011) Education in palliative and end-of-life care. The EPEC Project.

22. Rady MY, Verheijde JL, McGregor J (2008) Organ Procurement After Cardiocirculatory Death: A Critical Analysis. J Intensive Care Med 23: 303-312.

23. Ysebaert D, Van Beeumen G, De Greef K, Squifflet JP, Detry O, et al. (2009) Organ Procurement After Euthanasia: Belgian Experience. Transplant Proc 41 585-586. 
Citation: Rady MY, Verheijde JL, Potts M (2011) Quality Palliative Care or Physician-assisted Death: A Comment on the French Perspective of Endof-life Care in Neurological Disorders. J Clinic Res Bioeth 2:102e. doi:10.4172/2155-9627.1000102e

Page 5 of 5

24. Vincent JL, Brimioulle S (2009) Non-Heart-Beating Donation: Ethical Aspects. Transplant Proc 41: 576-578.

25. Wilkinson D, Savulescu J (in press) Should we allow organ donation euthanasia? Alternatives for maximizing the number and quality of organs for transplantation. Bioethics.
26. Auyong DB, Klein SM, Gan TJ, Roche AM, Olson D, et al. (2010) Processed Electroencephalogram During Donation After Cardiac Death. Anesth Analg 110: $1428-1432$

27. Rady MY, Verheijde JL (2010) General Anesthesia for Surgical Procurement in Non-Heart-Beating Organ Donation: Why We Should Care. Anesth Analg 111: 1562. 